\title{
Evaluation of Kinesio Taping Applied to the Equine Thoracolumbar Spine: Clinical Response and Mechanical Nociceptive Threshold
}

\author{
Marta García Piqueres* • Paloma Forés Jackson
}

Received: 04 July 2021 | Accepted: 24 August 2021 | Published online: 28 August 2021

\begin{abstract}
Department of Animal Medicine and Surgery, Faculty of Veterinary Medicine, Universidad Complutense de Madrid 28040. Madrid, Spain.
\end{abstract}

\section{Correspondence} Marta García Piqueres Email:

martagpiqueres@hotmail.com

\begin{abstract}
Kinesio taping (KT) is a technique extrapolated from human physiotherapy consisting of the application of an elastic tape to the skin to trigger analgesic, muscular, postural correction and circulatory effects. It is an easily applicable technique that has been developed in the field of equine physiotherapy over the last decade. The objective of this research is to evaluate the analgesic effect of KT applied to spinous processes of the horse measuring mechanical nociceptive threshold (MNT). KT was applied on 5 spinous processes of 15 horses, in two different experiments, comprising KT with 50\% tension (KTT) and KT with no tension (KTNT). Measurements were taken before application of the tape (M0), 60 minutes after (M1) and 24 hours after, following its removal (M2). Clinical assessment of sensitivity to palpation was conducted at M0 and M2. Outcomes obtained at $\mathrm{M} 0$ were compared to those obtained at $\mathrm{M} 1$ and $\mathrm{M} 2$, and between both tests (KTT-KTNT). A significant increase in the MNTs at M1 was observed in both tests but not maintained following its removal 24 hours later. Sensitivity to palpation decreased in practically all the spinous processes in both tests. No significant changes were observed in the comparative analysis between both tests. KT applied to spinous processes of the horse with and without tension causes an increase in the MNTs 60 minutes after application. This effect is not sustained following taping removal although there is a clinically significant decrease of the sensitivity to palpation of the spinous processes.
\end{abstract}

Keywords Back, Horse, Kinesio tape, Pressure algometry

\section{Introduction}

The horse's spine is a fundamental structure within its biomechanics that limits the animal's functional capacity when it presents with pain, clinically manifesting as a reduction of sports performance and as behavioural alterations (Allen et al. 2010).

The prevalence of back pathologies involving the spine is high, with large age distribution (5-22 years)
(Mayaki et al. 2019) and the majority of these lesions are found in the spinous process (kissing spines) (Jeffcott 1980), most commonly in the middle and caudal thoracic regions (T13-T18) (Clayton and Stubbs 2016).

Diagnosis is often boring due to the difficulty of objectively evaluating the degree of pain the horse is experiencing (Burns et al. 2018). 
Algometry is a tool that enables sensitivity quantification at a specific point through the application of constant and progressive pressure to the skin until a painful response is obtained. The pressure achieved in this moment is the minimum needed to induce a painful response (mechanical nociceptive threshold, MNT) (Fischer 1987). The clinical improvement resulting from the decrease in pain translates into increases in the nociceptive threshold (Fischer 1986). The algometer is a non-invasive, objective, reliable method that is well-tolerated by horses when quantifying musculoskeletal pain in the neck and spine, making it possible to evaluate the evolution of treatments and their outcomes (Haussler and Erb 2006a, Menke et al. 2016). It has also demonstrated a good correlation to clinical findings (Varcoe-Cocks et al. 2006).

The treatment of spinal pathologies has traditionally involved the systemic and local administration of antiinflammatories and muscle relaxants, combined in some cases with surgical interference (Garcia-Lopez 2018). The combination of these treatments with physiotherapy, results in an improvement of pain and functional recovery through the use of manual therapies, electrotherapy and therapeutic exercise (Bromiley 1999).

Kinesio taping (KT) is a technique developed by a Japanese chiropractor, Dr. Kenzo Kase, widely used in human physiotherapy, consisting of the application of an elastic tape to the skin, capable of acting on its mechanoreceptors to generate analgesic, muscular and circulatory effects in addition to postural correction (Kase et al. 2003; Molle 2016). Its effects depend on the way it is attached to the skin; in the "space-correction technique", the elevation of the tissue triggered by the taping (convolutions) decreases the pressure on the nociceptors and increases blood circulation, providing analgesia (Kase et al. 2003).

The exact mechanism by which kinesio taping works has not been determined, but some authors indicate that this analgesic effect responds to the "Gate Control" theory (Melzack and Wall 1965), according to which the taping stimulates the fast-conducting myelinated nerve fibers (A-beta), the information of which is prioritised over the painful stimulus on a spinal level (Castro-Sanchez et al. 2012; Chang et al. 2018; Paoloni et al. 2011).
It is a low-cost technique that is lacking in complications and easy to apply which has proven its analgesic efficacy in humans although there is no evidence that its effects are prolonged (Thelen et al. 2008; Kalron and Bar-Sela 2013; Artioli and Bertolini 2014).

Kinesio taping has been extensively developed in the world of equine physiotherapy over the last decade, manly applied for pain control, for the release of fascial restrictions, to increase muscle tone or to decrease muscle stiffness or for lymphatic drainage, among other applications. Nonetheless, there remains a lack of scientific studies demonstrating its effects on horses.

The objective of the current is to evaluate the analgesic effect of kinesio taping, with and without tension, on the horse's spinous process. There have not been any studies on the analgesic effects of this therapy on the horse back to date. Our hypothesis maintains that kinesio taping applied using the spacecorrection lifting technique over the spinous process of horses ridden regularly leads to an increase in the MNTs (analgesic effect).

\section{Materials and Methods}

\subsection{Ethics}

Employed horses were client-owned. Owners have signed an informed consent. Information about horses was kept confidential.

\subsection{Horses}

This study included fifteen client-owned horses, 5 mares, 8 geldings and 2 stallions, aged between 7 and 22 years old (mean age of 15.5 years) and stabled in four equestrian centres located in the same area. All horses did moderate physical activity in the dressage and show jumping categories. Inclusion criteria wasn't based on age or type of physical activity because back disorders have been described in horses of a large range of age and all kind of equestrian disciplines (Jeffcott 1980; Mayaki et al. 2019). A complete medical history was taken before inclusion of the horses in the study.

Horse selection was based on the following inclusion criteria: 1) horses doing physical activity with ridden work at least three times per week, 2) hair length on back not greater than $1 \mathrm{~cm}, 3$ ) not having received medical treatments or physiotherapy on the back in the two months prior to the trial, 4) not having received medication via any mode of administration. 
Selection of ridden horses was made based on the criteria of other authors that that assume that ridden horses are prone to low-grade or subclinical back disorders (Sullivan et al. 2008). The trial was conducted in the summer period to facilitate the inclusion of horses with short hair.

\subsection{Experimental procedure}

The experimental design of this randomised crossover study comprises evaluation of the effects of kinesio taping over 3 thoracic and 2 lumbar spinous processes (T12, T15, T18, L2 and L4), which were located using an ultrasound (EPTE Ultrasound wifi eco1, Ionclinics and Deionic SL, Spain) (Fig. 1) and marked with a skin marker.

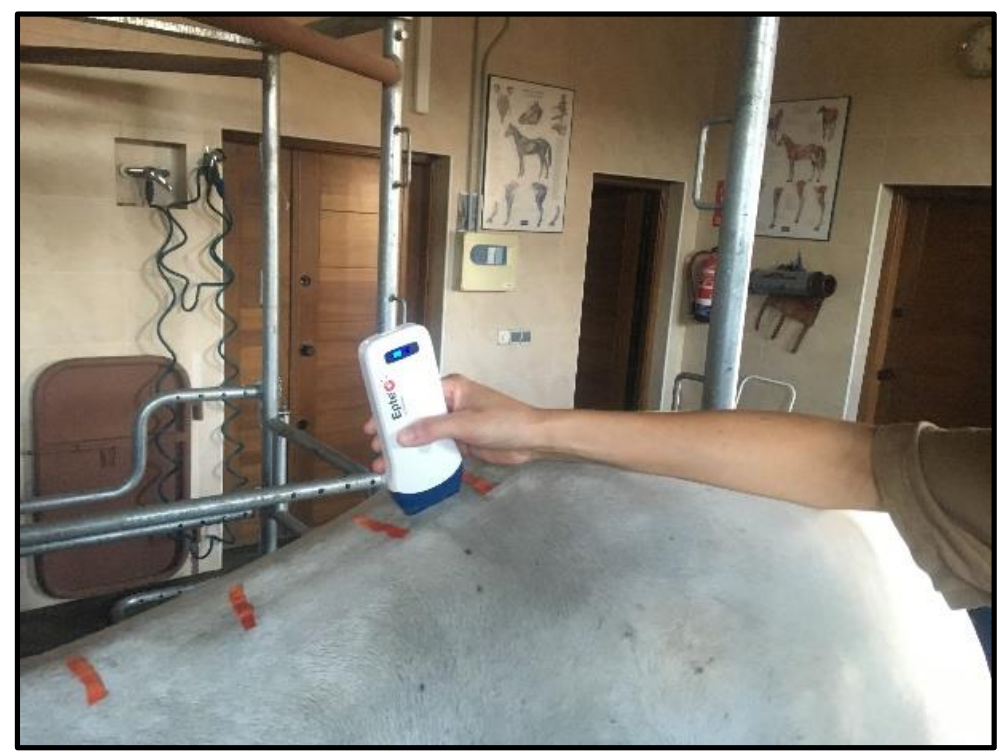

Fig. 1. Ultrasound guided localization of spinous processes.

All 15 horses were randomly assigned (Research Randomizer) the kinesio taping application with tension (KTT test) or without tension (KTNT test), and 24 hours after completion were subjected to the remaining test; thus all horses participated in both tests (Fig. 2). MNT measurements were taken before application of the tape (basal moment, M0), 60 minutes following application (moment 1, M1) and 24 hours following application, after its removal (moment 2, M2). The sensitivity to palpation of the spinous processes prior to tape application (M0) and following removal of the same (M2) was evaluated and classified as grade 0 (no pain), grade 1 (mild pain), grade 2 (moderate pain) or grade 3 (severe pain), based on the adapted De Heus scale (De Heus et al. 2010).

\subsection{MNT measurements}

Measurements of the nociceptive threshold were taken using a digital algometer (ProdPlus Algometer, Topcat Metrology Ltd, UK) calibrated in a range of 0.5 to 25 Newtons $(\mathrm{N})$, with a metal tip with a diameter of $2 \mathrm{~mm}\left(0.031 \mathrm{~cm}^{2}\right)$. Measurements were taken by placing the algometer perpendicular to the skin, applying a pressure of $2 \mathrm{~N} / \mathrm{sec}$, until the horse showed at least one sign of having reached its pain threshold: skin fasciculation, muscle contraction, extension of the back, shying away from the operator (Haussler and Erb 2006a; Menke et al. 2016). Prior to the start of the study, the horses had been made accustomed to having algometer measurements taken.

The points of measurement were marked on the skin and the tape using a marker. 3 measurements were taken at each spinous process, with a 30 second interval between measurements (Fig. 3). The measurement order of each point was randomised in each horse. The operator, with clinical experience in back palpation (M.G.P.), remained blind to the measurement value until completion of the same, and did not know which horse belonged to each group at the time of assessment. 


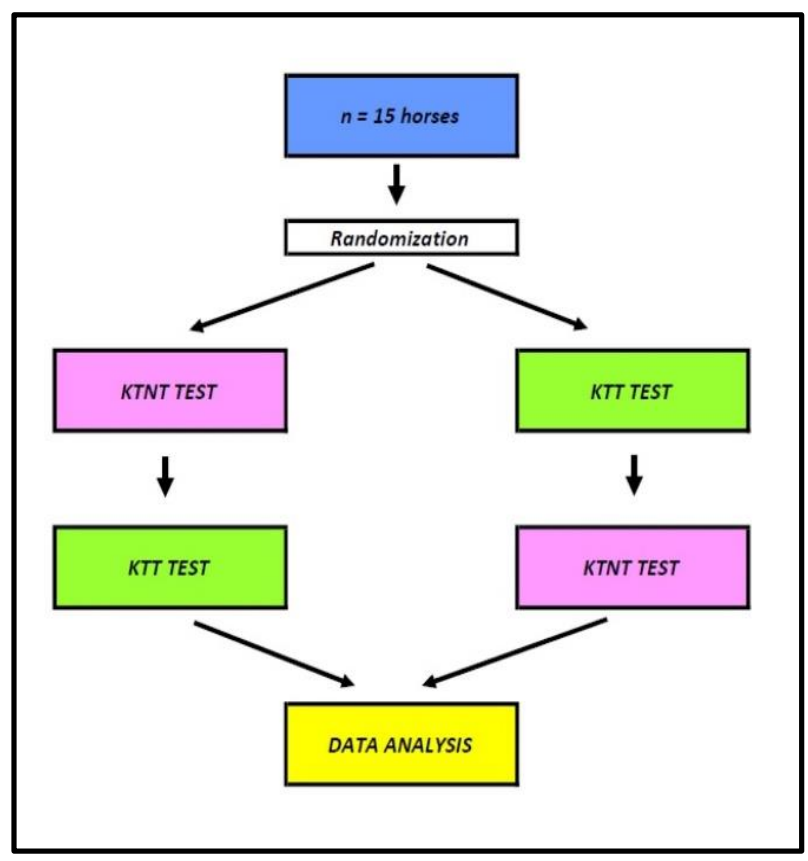

Fig. 2. Flow chart of the study.

\subsection{Kinesio taping application}

Five strips of orange-coloured Kinesio tape (VetkinTape ${ }^{\circ}$, Thyson Group B. V., Netherlands) were used, measuring $6 \mathrm{~cm}$ in width and $30 \mathrm{~cm}$ in length. The characteristics of this tape are its longitudinal elasticity and adhesiveness (alcohol-free adhesive acrylic layer), it is made of cotton (92\%), is water-resistant, breathable and has properties comparable to those of the human epidermis (thickness, elasticity, weight) [24, information provided by Vetkin Tape ${ }^{\circledR}$ ]. This kind of tape is specially designed for horses with a stronger glue that ensures stickiness on the hairy skin. Horses weren't clipped to mimic the conditions of clinical use. The tape edges were rounded to prevent it from unsticking early on. The $10 \mathrm{~cm}$ at each end were considered anchor points, while the central $10 \mathrm{~cm}$ acted as the treatment zone.

The horses were taped according to the "space correction lifting" technique described by Dr. Kenzo Kase (Fig 4).

Application without tension was administered by sticking the tape transversely onto the spinous process without tensing it. Application with tension was also administered transversely on the process but stretching the central part of the tape to $50 \%$ of its basal length, while also stretching the horse's skin

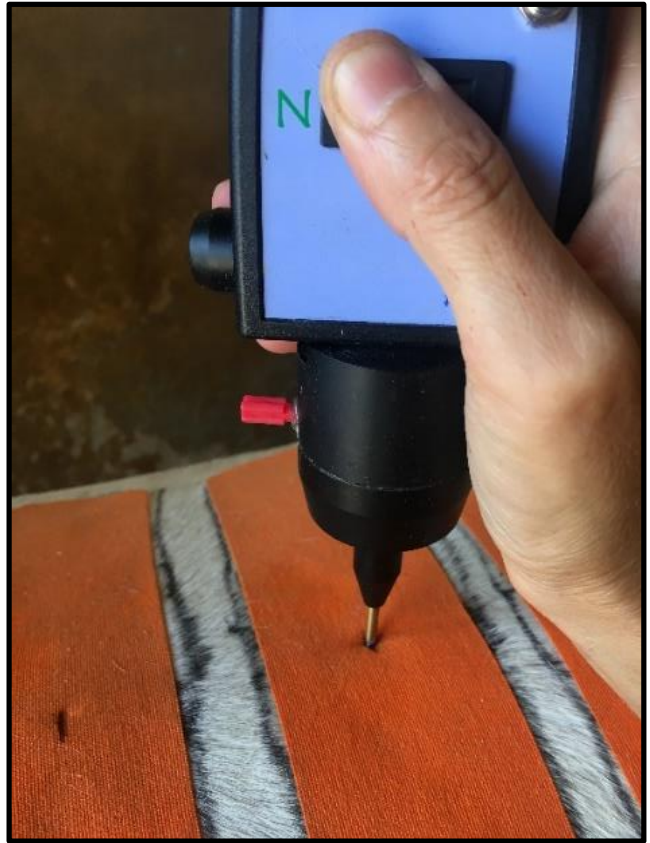

Fig. 3. Algometer measurements of the MNTs

through the thoracic flexion reflex. The skin of the horse was marked over the spinous process with two lines separated $15 \mathrm{~cm}$ in order to stretch the tape exactly $50 \%$ (from $10 \mathrm{~cm}$ to $15 \mathrm{~cm}$ ).

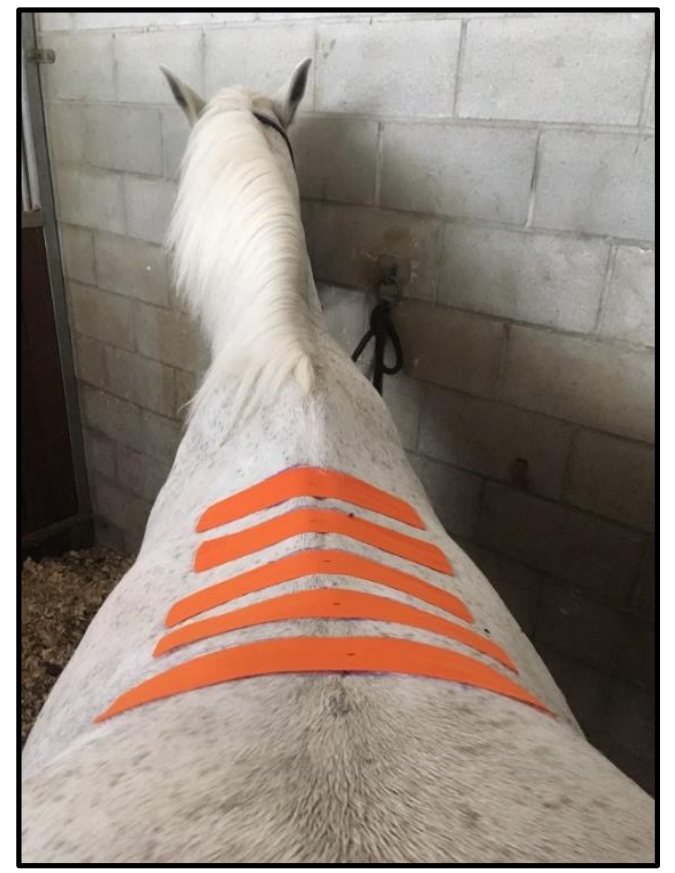

Fig. 4. Kinesio taping application over the spinous processes. 


\subsection{Statistical analysis}

To evaluate the repeatability of the results obtained using the digital algometer in the three measurements taken at each point, the Intraclass Correlation Coefficient (ICC) was calculated, by using a mixed model with random effects for average measurements and total agreement $(\operatorname{ICC}(3, \mathrm{k})$ two-way mixed average measures, consistency / absolute agreement). Adaptation or sensitisation phenomena were assessed by ANOVA.

Variables included in the statistical model were MNT and sensitivity to palpation of the spinous processes (pain scale).The differences between the different moments of mechanical nociceptive threshold (MNT's) measurement were evaluated in a Student's t-test for paired samples or a Signed Rank Wilcoxon test as dictated by the data normality in each case (evaluated using the Kolmogorov-Smirnov test). After comparing moments, the p-values were adjusted in each case by applying the Bonferroni Correction. Pain scale outcomes were evaluated in a Signed Rank Wilcoxon test. Values of $\mathrm{p}<0.05$ were considered statistically significant. Software used for the statistical analysis was SPSS v25 and SAS v9.4.

\section{Results}

\subsection{Repeatability}

In all measurements taken in the KTNT test the ICC was greater than to 0.9, while in the case of the KTT test the ICC values were slightly lower but in no case below 0.82. According to Landis and Koch [25], a close-to-perfect agreement is demonstrated when the ICC value is between 0.81 and 1 , meaning our ICC values indicate a good repeatability of the algometer measurements (Table 1).

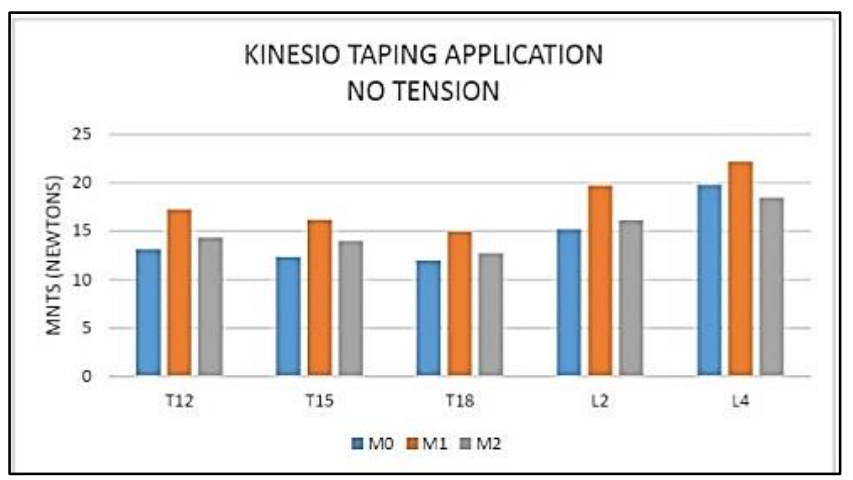

\subsection{Adaptation / sensitization to the algometer measurements}

After conducting the ANOVA test to assess the different measurements taken with the algometer at each point and in each moment, no statistically significant increasing or decreasing trend of these successive values was observed. The $\mathrm{p}$ value is considerably greater than 0.05 in all cases; the only $p$ value below 0.044 is detected in the KTT test in the M0 measurements of the thoracic process 12. The rest of the $\mathrm{p}$ values do not present a statistically significant trend towards either accommodation or sensitisation in the successive measurements of any single spinous process.

\subsection{Clinical evaluation}

Clinical evaluation using finger pressure comprises 75 measurements ( 5 spinous processes of each one of the 15 horses included in the study). In the previous evaluation (M0) of the KTNT test, $78 \%$ of the tests showed no evidence of pain; of the remaining $22 \%$, pain was only moderate in one of them, while it was mild in the rest. In the clinical evaluation conducted in M2, mild pain was only detected in one test, the rest were pain-free $(98.6 \%)$. In the previous evaluation (M0) of the KTT test, $80 \%$ of the evaluations showed no evidence of pain; in M2, 96\% of the measurements were pain-free (Table 2).

\subsection{MNT values}

The mean basal MNT values in M0 are similar in both tests and in all horses were higher in the most caudal vertebrae (L2 and L4) compared to the most cranial vertebrae (T12, T15 and T18) (Fig. 5).

Fig. 5. Mechanical nociceptive thresholds (MNTs) means for each spinous process, measured before kinesio taping application (M0), 60 minutes after (M1), and 24 hours after, once removed (M2), for both tests (50\% tension and no tension). 
Table 1. Intraclass Correlation Coefficients (icc). ICC's were calculated for each MNT measurement, before kinesio taping application (M0), one hour after kinesio taping application (M1) and 24 hours after kinesio taping application, once it was

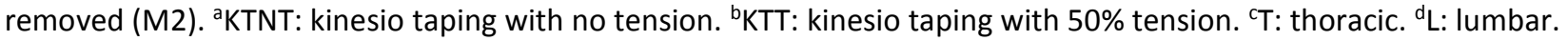

\begin{tabular}{cccc}
\hline KTNT $^{\mathrm{a}}$ & $\mathrm{M} 0$ & $\mathrm{M} 1$ & $\mathrm{M} 2$ \\
\hline${ }^{\mathrm{C}} 12$ & 0.965 & 0.941 & 0.968 \\
\hline $\mathrm{T} 15$ & 0.961 & 0.918 & 0.960 \\
\hline $\mathrm{T} 18$ & 0.906 & 0.940 & 0.952 \\
\hline${ }^{\mathrm{d} L 2}$ & 0.940 & 0.919 & 0.947 \\
\hline L4 & 0.933 & 0.936 & 0.980 \\
\hline KTT $^{\mathrm{b}}$ & $\mathrm{M} 0$ & $\mathrm{M} 1$ & $\mathrm{M} 2$ \\
\hline $\mathrm{T}^{\mathrm{T}}$ & 0.854 & 0.824 & 0.916 \\
\hline $\mathrm{T} 15$ & 0.937 & 0.909 & $0 ., 899$ \\
\hline $\mathrm{T} 18$ & 0.857 & 0.898 & 0.929 \\
\hline L2 & 0.947 & 0.959 & 0.935 \\
\hline L4 & 0.959 & 0.958 & 0.962 \\
\hline & & &
\end{tabular}

Table 2. Pain scoring outcomes (clinical palpation of spinous processes). Pain was measured by palpation before kinesio taping application (M0) and 24 hours after kinesio taping application, once removed (M2). ${ }^{a}$ number of horses (\%) ${ }^{b}$ KTNT: kinesio taping with no tension ${ }^{\mathrm{C}} \mathrm{KTT}$ : kinesio taping tension $50 \%$.

\begin{tabular}{|c|c|c|c|c|c|c|c|c|}
\hline \multirow[b]{2}{*}{ Site } & \multicolumn{4}{|c|}{ MO } & \multicolumn{4}{|c|}{ M2 } \\
\hline & Painfree & Mild & Moderate & Severe & Painfree & Mild & Moderate & Severe \\
\hline$T 12$ & $9(60)^{a}$ & $5(33)$ & $1(7)$ & 0 & $14(93)$ & $1(7)$ & 0 & 0 \\
\hline \multirow{4}{*}{$K T N T^{b}$} & $10(67)$ & $5(33)$ & 0 & 0 & $15(100)$ & 0 & 0 & 0 \\
\hline & $12(80)$ & $3(20)$ & 0 & 0 & $15(100)$ & 0 & 0 & 0 \\
\hline & $14(93)$ & $1(7)$ & 0 & 0 & $15(100)$ & 0 & 0 & 0 \\
\hline & $14(93)$ & $1(7)$ & 0 & 0 & $15(100)$ & 0 & 0 & 0 \\
\hline$T 12$ & $9(60)$ & $6(40)$ & 0 & 0 & $14(93)$ & $1(7)$ & 0 & 0 \\
\hline$T 15$ & $10(67)$ & $5(33)$ & 0 & 0 & $14(93)$ & $1(7)$ & 0 & 0 \\
\hline $\mathrm{KTT}^{\mathrm{c}}$ & $12(80)$ & $2(13)$ & $1(7)$ & 0 & $14(93)$ & $1(7)$ & 0 & 0 \\
\hline$L 2$ & $14(93)$ & $1(7)$ & 0 & 0 & $15(100)$ & 0 & 0 & 0 \\
\hline$L 4$ & $15(100)$ & 0 & 0 & 0 & $15(100)$ & 0 & 0 & 0 \\
\hline
\end{tabular}

\section{- M0-M1 Comparison}

In the KTNT test, when the basal MNTs (M0) were compared with those obtained in M1, an increase in all localisations was observed, though it was most apparent in T12 and L2 (means of 4.12 and 4.49, p values of 0.007 and p 0.003 respectively). In the KTT test, an increase in the MNTs was also observed in all mean values obtained but was not as pronounced as in the KTNT test; the biggest increase was obtained at $\mathrm{L} 2$ (mean of $3.05 \mathrm{n}$ ). The $\mathrm{p}$ value in these cases is also $<0.05$ except at T18 (mean $1.27 \mathrm{~N}, \mathrm{p} 0.2$ ) and at L4 (mean $0.96 \mathrm{~N}, \mathrm{p} 0.5$ ).

Comparing the mean MNT increases obtained between M0 and M1, the variations were limited and present a high $\mathrm{p}$ value $(\mathrm{p}>0.05)$ in all localisations (Table 3A).

\section{- M0-M2 Comparison}

In both tests, the variations in the mean MNT values between M0 and M2 are not noticeable (below $2 \mathrm{~N}$ in all the processes evaluated).

In the KTNT test, the highest mean value between M0 and M2 occurred at T15 (p 0.03). The mean value at L4 is negative $(-1.3 \mathrm{~N})$, implying an increase in sensitivity rather than a decrease.

In the KTT test, the increases in the MNTs between $\mathrm{M} 0$ and M2 are lower than in the KTNT test, and in all cases below $1 \mathrm{~N}(\mathrm{p}>0.05)$. The trend at $\mathrm{L} 4$ to generate a negative value (mean $-2.51 \mathrm{~N}, \mathrm{p} 0.006$ ) persisted.

When the mean MNT differences between M0-M2 of both tests were compared, slightly higher values were obtained in the KTNT test, but with low statistical significance ( $p>0.05$ in all cases) (Table 3B). 
Table 3. Mean MNT's differences between measures for each test.

A. Differences between mean MNT values (Newtons) evaluated before kinesio taping application (M0) and 60 minutes after application (M1), for each test (KTNT and KTT) and between both test (Comparison KTNT-KTT).

\begin{tabular}{lcccccc}
\hline & \multicolumn{2}{c}{ KTNT } & \multicolumn{2}{c}{ KTT } & \multicolumn{2}{c}{ Comparison KTNT-KTT } \\
& Mean difference & & Mean difference & & \multicolumn{2}{c}{ Mean difference } \\
M1-M0 & P value & M1-M0 & P value & M1-M0 & P value \\
\hline T12 & $4.12(4.52)^{a}$ & 0.007 & $2.43(1.4)$ & 0.0002 & $1.68(5.08)$ & 0.4 \\
\hline T15 & $3.84(2.98)$ & 0.0004 & $2.37(2.39)$ & 0.004 & $1.47(4.11)$ & 0.4 \\
\hline T18 & $2.97(3.09)$ & 0.002 & $1.27(2.66)$ & 0.2 & $1.7(3.62)$ & 0.2 \\
\hline L2 & $4.49(4.44)$ & 0.003 & $3.05(4.08)$ & 0.02 & $1.44(5.81)$ & 0.7 \\
\hline L4 & $2.44(3.54)$ & 0.04 & $0.96(2.98)$ & 0.5 & $1.47(4.42)$ & 0.4 \\
\hline
\end{tabular}

B. Differences between mean MNT values (Newtons) evaluated before kinesio taping application (M0) and 24 hours after application, once removed (M2), for each test (KTNT and KTT) and between both test (comparison KTNT-KTT). ${ }^{\mathrm{a} M e a n}$ (SD).

\begin{tabular}{lcccccc}
\hline & \multicolumn{2}{c}{ KTNT } & \multicolumn{2}{c}{ KTT } & \multicolumn{2}{c}{ Comparison KTNT-KTT } \\
& Mean difference & & Mean difference & Mean difference \\
M2-M0 & P value & M2-M0 & P value & M2-M0 & P value \\
\hline T12 & $1.21(2.96)$ & 0.3 & $0.86(2.9)$ & 0.5 & $0.35(3.83)$ & 1 \\
\hline T15 & $1.68(2.35)$ & 0.03 & $0.15(3.3)$ & 1 & $1.53(3.07)$ & 0.1 \\
\hline T18 & $0.74(2.74)$ & 1 & $0.24(2.8)$ & 1 & 1 & $0.49(3.43)$ \\
\hline L2 & $0.9(2.97)$ & 0.5 & $0.7(4.27)$ & 1 & $0.19(4.49)$ & $1.21(3.91)$ \\
\hline L4 & $-1.3(4.71)$ & 0.8 & $-2.51(2.75)$ & 0.006 & 0.5 \\
\hline
\end{tabular}

\subsection{Tolerance to procedure}

Following some algometer measurements, small depressions in the skin were observed in the zones where it had come in contact with the tip. These did not result in lesions and disappeared after a few minutes.

Removal of the tape did not result in signs of skin alterations in any horse. In the KTNT test, 24 hours following adhesion, the tape remained in good condition in all horses; in the KTT test, $53 \%$ of the horses presented some unstuck anchor points, while the central part stuck to the spinous process remained intact in all horses.

\section{Discussion}

The use of kinesio taping in the field of equine physiotherapy has become widespread in recent years, yet there is a significant gap in the clinical research into its effects on animals. Nowadays, the way the technique is applied on horses comes from the original development of kinesio taping for human beings and the research done by human therapists. Nonetheless, the results obtained in humans cannot be extrapolated directly to the veterinary setting without taking into consideration the fact that the presence of hair may be a limiting factor of its benefits. No articles have been published, to the best of the authors' knowledge, on the analgesic effect of kinesio taping in horses.

The obtained results indicate good repeatability of the algometry technique with ICCs of over 0.82 in all evaluations. This repeatability, which has already been demonstrated in other studies with horses (Chambers et al. 1990; Haussler and Erb 2006a; Haussler et al. 2008), is attained with just one operator, taking three measurements at each point, with a constant pressure rate (Nussbaum and Downes 1998). The importance of maintaining constant pressure is due to the linear relationship existing between said rate and the nociceptive threshold value (List et al. 1991).

We did not observe any accommodation or sensitisation phenomena in successive measurements as reported by other authors (Haussler and Erb 2006a) associating this effect with the frequency of the same (every 3-4 seconds); we included a 30second pause between them and this could explain the absence of these phenomena.

The obtained demonstrate analgesia in the algometer measurement 60 minutes after the kinesio tape application, in both tests (KTT and KTNT), but with higher mean increases in the MNT values in the group without tension. For the measurements taken at M1 
the algometer was placed against the adhered tape; to evaluate whether this triggered an alteration in the MNT values, a previous experiment with different horses was conducted, evaluating the same point with and without adhered kinesio tape, and no differences were observed, concluding that the presence of the tape did not affect the MNT value.

It was observed that the MNT increases at 60' after tape application did not maintain the same relevance in the 24 hours following removal of the same, meaning the analgesic effect, measured by pressure algometer, is not sustained. In humans, some studies only detect short-term improvements (Thelen et al. 2008; Paoloni et al. 2011) and while in these the subjects were taped for longer time periods, we do not believe the taping time is a key factor as an analgesic effect has also been demonstrated in patients with lower back pain after both 24 hours of taping (Chang et al. 2018) and after 45 minutes (Celenay and Kaya 2019).

The pronounced variability of the MNTs between individual MNTs (Vanderweeën et al. 1996; Haussler and Erb 2003; De Heus et al. 2010) renders it difficult to establish an absolute reference value below which the horse can be considered to feel pain. Nonetheless, differences of over $1 \mathrm{~kg}$ (surface area of the tip $1 \mathrm{~cm}^{2}$ ) between one zone and its contralateral (Haussler and Erb 2006b) are described as significant, and in horses who present musculoskeletal pathology the MNTs in these damaged zones are referred to as being less than $5 \mathrm{~kg} / \mathrm{cm}^{2}$ (surface area of the tip $1 \mathrm{~cm}^{2}$ ) (Haussler and Erb 2003).

It is important to note that it is not possible to compare algometry studies with tips presenting different surface areas and shapes as they produce a different distortion of the tissue, and though the bigger the tip surface the higher the MNT, this relationship is not linear (Taylor and Dixon 2012; Taylor et al. 2016). As described in other studies, in our sample we observed an increased MNT in the more caudal measurements compared to the more cranial, associated with a lower nociceptive density in the caudal region (Haussler and Erb 2006a) and an increase in the tissue thickness in the lumbar back zone compared to the thoracic zone (Pongratz and Licka 2017).

In the clinical evaluation outcomes analysis considerable improvements were obtained in M2 palpation compared to $\mathrm{M} 0$, in both tests, in those spinous processes presenting pain.

Two hypotheses could justify the difference between the clinical and algometric outcomes at M2. The first resides in the fact that horses without any recent history of back pain were chosen, the majority of which did not present painful spinous processes $(33 \%$ of the horses did not present pain in any of them), meaning the expected MNT variations are not very significant as there had not been any previous pain. The MNT improvement detected in M1 in both tests could respond to the analgesic effect of the taping that would diminish on removal of the same (M2), only persisting on those processes that clinically presented with pain in M0.

The difficulty of finding a homogeneous sample of horses with painful spinous processes led us to use animals that were frequently ridden, following the criteria of other authors (Sullivan et al. 2008) who assume that ridden horses experience subclinical back pain. Additionally, given that higher MNTs are described in ridden animals compared to those not ridden (Haussler and Erb 2006a), it was necessary to select only ridden horses to avoid any bias.

The second hypothesis to explain the variation in the outcomes obtained in M2 is based on the difference in the pressure applied to the spinous processes in both methods (algometry and palpation). While the pressure applied by a thumb in a palpation is described as being around $0.4-0.6 \mathrm{kgf} / \mathrm{cm}^{2}$ [17], the lowest MNT detected during our experimental procedure was $14.28 \mathrm{kgf} / \mathrm{cm}^{2}(4.4 \mathrm{~N}$, tip with a diameter of $2 \mathrm{~mm}$ ), meaning the maximum force applied during the clinical evaluation would be 30 times lower than that used by the algometer to generate a painful response in the most sensitive spinous process of the entire study.

Furthermore, if we take into consideration that tips with smaller surface areas give rise to more consistent and repeatable outcomes (Taylor et al. 2016), but cause less distortion of the deep tissues (Treede et al. 2002), the thumb would be generating less pressure and affecting deeper tissues than if were to use the tip of our algometer $\left(0.031 \mathrm{~cm}^{2}\right)$, which could explain the difference between the outcomes obtained with each method.

A collation of the tests (with and without tension) showed the differences between the mean values in 
the two comparisons (M0-M1 and M0-M2) are not pronounced $(<2 \mathrm{~N}$ in all cases) and they have high $\mathrm{p}$ values $(p>0.05)$. Hence, the outcomes of our study indicate that tensing the tape before applying it (50\% stretching) does not result in a greater analgesic benefit compared to not doing so, in line with other studies in humans which observed a similar analgesic improvement in applications with and without tension (Thelen et al. 2008; Chang et al. 2012; Silva Parreira et al. 2014; Macedo et al. 2019).

Based on the hypotheses put forward in the field of human physiotherapy, and eliminating a possible placebo effect not applicable in animals, we suggest that the taping generates a stimulus on the cutaneous mechanoreceptors capable of triggering pain inhibition mechanisms, as explained by the "Gate Control" theory put forward by Melzack and Wall (Thelen et al. 2008; Paoloni et al. 2011; Macedo et al. 2019). This theory claims that the cutaneous stimulus activates high-speed nerve pathways whose entrance into the central nervous system is prioritised over the painful stimulus conduction that is transmitted via slow nerve fibres. Hence, the stimulus triggered by the taping on the skin (regardless of its tension) could be sufficient to generate the analgesic response (Velasco-Roldan et al. 2018), which in turn would decrease once it had been removed, as we verified in the algometer measurement in M2.

A further hypothesis on its effects, proposed by the inventor of the technique (Kase et al. 2003), is based on the elevation of the skin caused by the taping following its application with tension, creating convolutions that decrease pressure on the nociceptors and improve blood flow in the affected area (Chang et al. 2012). In our outcomes, we did not obtain a significant difference between application with or without tension, but some studies in humans do describe improvements when the taping is applied by generating convolutions (Castro-Sanchez et al. 2012).

Regarding the percentage of tension, our choice, which may have impacted the outcomes, was based on Dr. Kenzo Kase's indications for the spacecorrection lifting technique, which recommends a tension of $25-50 \%$. Although some texts described the tension percentage according to the maximum length of the tensed tape, we measured it according to its basal length without tension, as recommended for techniques applied with a tension equal to or over $50 \%$ (Golab et al. 2017).
No skin reaction was observed in the horses following removal of the tape. Reports of allergic reactions post kinesio taping application are limited and improve without treatment once the tape has been removed (Chang et al. 2018).

One of the limitations of this study may have been the presence of hair; although we used a kind of tape specially designed for horses, the tape was stuck onto hair and not directly onto skin as recommended by the creator of the technique (Kase 2000; Kase et al. 2003). Other limiting factors were the fact that the horses did not present pain in all the spinous processes measured, that we did not have a control group in which taping was not applied and that the measurements were taken in a short time frame following one sole application.

\section{Conclusion}

The endpoint of this study was to evaluate the analgesic effect of kinesio taping on the horse's spine and we can conclude that its application, with both $50 \%$ tension and without tension, on the horse's spinous processes causes an elevation of the MNTs after 60 minutes. The increase in the MNTs is not maintained 24 hours after removal but a clinical improvement is detected in the palpation of the processes that presented with pain, in both tests (KTT y KTNT).

Kinesio taping is a simple technique to apply that complements medical and physiotherapeutic treatments of the back, and it has demonstrated its short-term analgesic effect.

\section{Acknowledgments}

The authors thank Dr. Polly M Taylor for the advice given on the algometer technique, Marc Van Zuilen for helping with the kinesio taping protocol and Ricardo García Mata for performing the statistical analysis of the data. We also wish to thank the horses and their owners for taking part on this study.

\section{Conflict of interest}

The authors declare no conflict of interest.

\section{References}

Allen KS, Johns S, Hyman M, Sislak S, Davis, Amory J (2010). How to diagnose and treat back pain in the horse. 56: 384-388. Proc. Am. Ass. Equine Practnrs.

Artioli DP, Bertolini GRF (2014). Kinesio taping: application and results on pain: systematic review (Kinesio taping: 
aplicação e seus resultados sobre a dor: revisão sistemática. Fisioterapia e Pesquisa, 21: 94-99.

Bromiley MW (1999). Physical therapy for the equine back. Veterinary Clinics of North America-Equine Practice, 15: 223.

Burns G, Dart A, Jeffcott L (2018). Clinical progress in the diagnosis of thoracolumbar problems inhorses. Equine Vet Education, 30: 477-485.

Castro-Sanchez AM, Lara-Palomo IC, Mataran-Penarrocha GA, Fernandez-Sanchez M, Sanchez-Labraca N, Arroyo-Morales M (2012). Kinesio Taping reduces disability and pain slightly in chronic non-specific low back pain: a randomised trial (58: 89, 2012). J Physiotherapy, 58: 143-143.

Celenay ST, Kaya DO (2019). Immediate effects of kinesio taping on pain and postural stability in patients with chronic low back pain. J Bodywork and Movement Therapies, 23: 206-210.

Chambers JP, Livingston A, Waterman AE (1990). A device for testing nociceptive thresholds in horses. J Ass Vet Anaesth., 17: 42-44.

Chang HY, Wang CH, Chou KY, Cheng SC (2012). Could Forearm Kinesio Taping Improve Strength, Force Sense, and Pain in Baseball Pitchers With Medial Epicondylitis? Clinical J Sport Med., 22: 327-333.

Chang NJ, Chou W, Hsiao PC, Chang WD, Lo YM (2018). Acute effects of Kinesio taping on pain, disability and back extensor muscle endurance in patients with low back pain caused by magnetic resonance imagingconfirmed lumbar disc degeneration. J Back and Musculoskeletal Rehabilitation, 31: 85-93.

Clayton HM, Stubbs NC (2016). Enthesophytosis and Impingement of the Dorsal Spinous Processes in the Equine Thoracolumbar Spine. J Equine Vet Sci., 47: 915.

De Heus P, Van Oossanen G, Van Dierendonck MC, Back W (2010). A Pressure algometer is a useful tool to objectively monitor the effect of diagnostic palpation by a physiotherapist in warmblood horses. J Equine Vet Sci., 30: 310-321.

Fischer AA (1986). Pressure threshold meter - its use for quantification of tender spots. Arch Physical Med and Rehabilitation, 67: 836-838.

Fischer AA (1987). Pressure algometry over normal muscles - standard values, validity and reproducibility of pressure threshold. Pain, 30: 115-126.

Garcia-Lopez JM (2018). Neck, back, and pelvic pain in sport horses. Vet Clinics North America-Equine Practice, 34: 235.

Golab A, Kulesa-Mrowiecka M, Golab M (2017). Comparative studies of physical properties of kinesiotapes. Bio-Medical Materials and Engineering, 28: 457-462.
Haussler KK, Behre TH, Hill AE (2008). Mechanical nociceptive thresholds within the pastern region of Tennessee Walking Horses. Equine Vet J., 40: 455-459.

Haussler KK, Erb HN (2003). Pressure algometry: objective assessment of back pain and effects of chiropractic treatment. 66-70. Lexington: American Assoc Equine Practitioners (AAEP).

Haussler KK, Erb HN (2006a) Mechanical nociceptive thresholds in the axial skeleton of horses. Equine Vet J., 38: 70-75.

Haussler KK, Erb HN (2006b). Pressure algometry for the detection of induced back pain in horses: a preliminary study. Equine Vet J., 38: 76-81.

Jeffcott LB (1980). Disorders of the thoracolumbar spine of the horse - A survey of 443 cases. Equine Vet J., 12: 197-210.

Kalron A, Bar-Sela S (2013). A systematic review, of the effectiveness of Kinesio taping (R) - Fact or fashion? European J Physical and Rehabilitation Med., 49: 699709.

Kase K (2000). Illustrated Kinesio taping. Tokyo, Japan: Ken Ikai Co LTD.

Kase K, Wallis J, Kase T (2003). Clinical therapeutic applications of the kinesio taping method. Tokyo, Japan: Ken Ikai Co LTD.

List T, Helkimo M, Karlsson R (1991). Influence of pressure rates on the reliability of a pressure threshold meter. Journal of craniomandibular disorders. facial \& oral pain, 5: 173-8.

Macedo LD, Richards J, Borges DT, Melo SA, Brasileiro JS (2019). Kinesio taping reduces pain and improves disability in low back pain patients: a randomised controlled trial. Physiotherapy, 105: 65-75.

Mayaki AM, Intan-Shameha AR, Noraniza MA, Mazlina M, Adamu L, Abdullah R (2019). Clinical investigation of back disorders in horses: A retrospective study (20022017). Vet World, 12: 377-381.

Melzack R, Wall PD (1965). Pain mechanisms - a new theory. Sci., 150: 971-979.

Menke ES, Blom G, van Loon J, Back W (2016). Pressure algometry in Icelandic horses: interexaminer and intraexaminer reliability. J Equine Vet Sci., 36: 26-31.

Molle S (2016). Kinesio taping fundamentals for the equine athlete. Vet Clinics North America-Equine Practice, 32: 103.

Nussbaum EL, Downes L (1998). Reliability of clinical pressure-pain algometric measurements obtained on consecutive days. Physical Therapy, 78: 160-169.

Paoloni M, Bernetti A, Fratocchi G, Mangone M, Parrinello L, Cooper MD, Sesto L, Di Sante L, Santilli V (2011). Kinesio taping applied to lumbar muscles influences clinical and electromyographic characteristics in chronic low back pain patients. European J Physical and Rehabilitation Med., 47: 237-244. 
Pongratz U, Licka T (2017). Algometry to measure pain threshold in the horse's back - An in vivo and in vitro study. Bmc Vet Res., 13.

Silva Parreira PdC, Menezes Costa LdC, Hespanhol LC, Lopes JrAD, Pena Costa LO (2014). Current evidence does not support the use of Kinesio Taping in clinical practice: a systematic review. J Physiotherapy, 60: 3139.

Sullivan KA, Hill AE, Haussler KK (2008). The effects of chiropractic, massage and phenylbutazone on spinal mechanical nociceptive thresholds in horses without clinical signs. Equine Vet J., 40: 14-20.

Taylor P, Dixon M (2012). A review of mechanical nociceptive threshold data in eight mammalian species. In Proceedings of the $14^{\text {th }}$ World congress on pain, 12. Milan, Italy.

Taylor PM, Crosignani N, Lopes C, Rosa AC, Luna SPL, Puoli JNP (2016). Mechanical nociceptive thresholds using four probe configurations in horses. Vet Anaes Analg., 43:99-108.

Thelen MD, Dauber JA, Stoneman PD (2008). The clinical efficacy of Kinesio tape for shoulder pain: A randomized, double-blinded, clinical trial. J Orthopaedic \& Sports Physical Therapy, 38: 389-395.
Treede RD, Rolke R, Andrews K, Magerl W (2002). Pain elicited by blunt pressure: neurobiological basis and clinical relevance. Pain, 98: 235-240.

Vanderweeën L, Oostendorp RAB, Vaes P, Duquet W (1996). Pressure algometry in manual therapy. Manual Therapy, 1: 258-265.

Varcoe-Cocks KK, Sagar N, Jeffcott LB, McGowan CM (2006). Pressure algometry to quantify muscle pain in racehorses with suspected sacroiliac dysfunction. Equine Vet J., 38: 558-562.

Velasco-Roldan 0 , Riquelme I, Ferragut-Garcias A, Heredia-Rizo AM, Rodriguez-Blanco C, Oliva-PascualVaca A (2018). Immediate and short-term effects of Kinesio taping tightness in mechanical low back pain: $A$ randomized controlled trial. Pm\&R, 10: 28-35.

How to cite this article: Piqueres MG, Jackson PF. Evaluation of kinesio taping applied to the equine thoracolumbar spine: clinical response and mechanical nociceptive threshold. J Vet Med Res., 2021; 28(1): 111. https://doi.org/10.21608/jvmr.2021.84001.1039 